EPJ Web of Conferences 116, 11005 (2016)

DOI: $10.1051 /$ epjconf/201611611005

(C) Owned by the authors, published by EDP Sciences, 2016

\title{
Baikal-GVD: Results, status and plans
}

A.D. Avrorin ${ }^{1}$, A.V. Avrorin 1 , V.M. Aynutdinov ${ }^{1}$, R. Bannash ${ }^{7}$, I.A. Belolaptikov ${ }^{2}$, D.Yu. Bogorodsky ${ }^{3}$, V.B. Brudanin ${ }^{2}$, N.M. Budnev ${ }^{3}$, I.A. Danilchenko ${ }^{1}$, G.V. Domogatsky ${ }^{1}$, A.A. Doroshenko ${ }^{1}$, A.N. Dyachok ${ }^{3}$, Zh.-A.M. Dzhilkibaev ${ }^{1}$, S.V. Fialkovsky ${ }^{5}$, A.R. Gafarov ${ }^{3}$, O.N. Gaponenko ${ }^{1}$, K.V. Golubkov ${ }^{1}$, T.I. Gress ${ }^{3}$, Z. Honz ${ }^{2}$, K.G. Kebkal ${ }^{7}$, O.G. Kebkal ${ }^{7}$, K.V. Konischev², A.V. Korobchenko ${ }^{2}$, A.P. Koshechkin ${ }^{1}$, F.K. Koshel ${ }^{1}$, A.V. Kozhin ${ }^{4}$, V.F. Kulepov ${ }^{5}$, D.A. Kuleshov ${ }^{1}$, V.I. Ljashuk ${ }^{1}$, M.B. Milenin ${ }^{5}$, R.A. Mirgazov ${ }^{3}$, E.R. Osipova ${ }^{4}$, A.I. Panfilov ${ }^{1}$, L.V. Pan'kov ${ }^{3}$, E.N. Pliskovsky², M.I. Rozanov ${ }^{6}$, E.V. Rjabov ${ }^{3}$, B.A. Shaybonov ${ }^{2}$, A.A. Sheifler ${ }^{1}$, M.D. Shelepov ${ }^{1}$, A.V. Skurihin ${ }^{4}$, A.A. Smagina ${ }^{2}$, O.V. Suvorova ${ }^{1}$, V.A. Tabolenko ${ }^{3}$, B.A. Tarashansky ${ }^{3}$, S.A. Yakovlev ${ }^{7}$, A.V. Zagorodnikov ${ }^{3}$, and V.L. Zurbanov ${ }^{3}$

${ }^{1}$ Institute for Nuclear Research, 60th October Anniversary pr. 7A, Moscow 117312, Russia

2 Joint Institute for Nuclear Research, Dubna 141980, Russia

${ }^{3}$ Irkutsk State University, Irkutsk 664003, Russia

${ }^{4}$ Skobeltsyn Institute of Nuclear Physics MSU, Moscow 119991, Russia

${ }^{5}$ Nizhni Novgorod State Technical University, Nizhni Novgorod 603950, Russia

${ }^{6}$ St. Petersburg State Marine University, St. Petersburg 190008, Russia

${ }^{7}$ EvoLogics GmbH, Berlin, Germany

\begin{abstract}
The future next-generation neutrino telescope Baikal-GVD will be a km3-scale array aimed at the detection of astrophysical neutrino fluxes. It will have modular structure and consist of functionally independent sub-arrays - clusters of strings of optical modules. The prototyping phase of the project has been concluded in 2015 with the deployment of the first cluster of Baikal-GVD in Lake Baikal. We discuss the current status and perspectives of the Baikal-GVD project.
\end{abstract}

\section{Introduction}

The next generation neutrino telescope Baikal-GVD is a kilometer-scale deep underwater Cherenkov detector currently under construction in Lake Baikal [1]. Baikal-GVD is designed to detect astrophysical neutrino fluxes at energies from a few $\mathrm{TeV}$ up to the highest energies of $10^{6} \mathrm{TeV}$ and particularly mapping the high-energy neutrino sky in the Southern Hemisphere including the region of the Galactic center.

The site chosen for the experiment is in the southern basin of Lake Baikal. Here, the combination of hydrological, hydro-physical, and landscape factors is optimal for deployment and operation of the neutrino telescope. The water depth is about $1360 \mathrm{~m}$ at distances beginning from about three kilometers

This is an Open Access article distributed under the terms of the Creative Commons Attribution License 4.0, which permits unrestricted use, distribution, and reproduction in any medium, provided the original work is properly cited. 

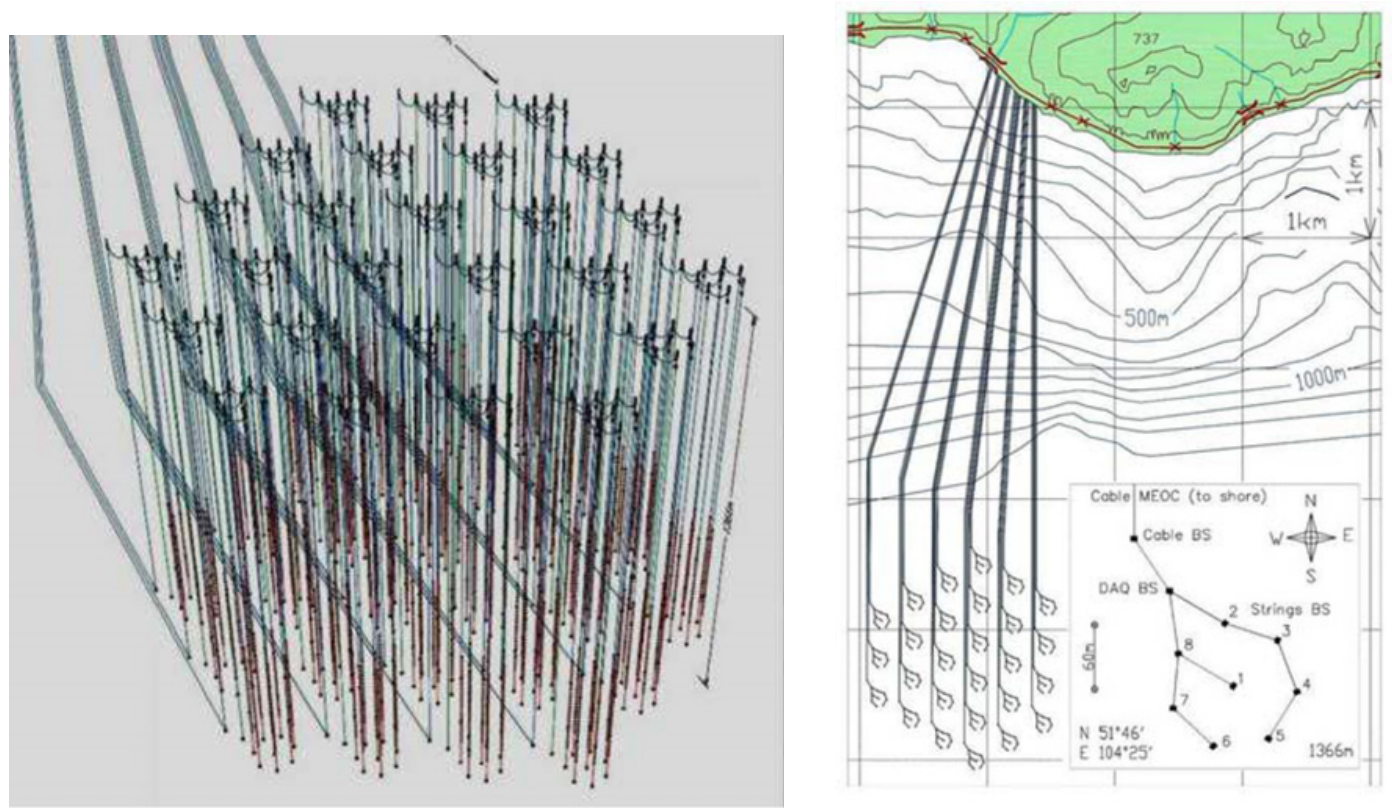

Figure 1. Left: Artist's view of the GVD-telescope. Right: Layout of GVD. Inner box: layout of a single GVD-cluster.

from the shore. The water transparency is characterized by an absorption length of about 20-25 $\mathrm{m}$ and a scattering length of 30-50 m [2]. The water luminescence is moderate at the detector site.

Baikal-GVD is a 3-dimensional lattice of optical modules (OMs) - photomultiplier tubes housed in transparent pressure spheres. The OMs are arranged at vertical load-carrying cables to form strings. The telescope has a modular structure and consists of clusters of strings - functionally independent sub-arrays, which are connected to shore by individual electro-optical cables (see Fig. 1).

The first prototype of the GVD electronics was tested in Lake Baikal in April 2008. It was a reducedsize section with six optical modules. This detection unit provided the possibility to study basic elements of the future detector: new optical modules and the Flash Analog-to-Digital Converter (FADC) based measuring system [3]. During the next 2 years different versions of prototype string were tested in Lake Baikal as a part of the old NT200+ detector. The operation of these prototype strings in 2009 and 2010 allowed a first assessment of the DAQ performance [4, 5].

The prototyping/early construction phase of the Baikal-GVD project, which aims at construction and operation of the first demonstration cluster of GVD, has been started in 2011 by deployment and operation of autonomous engineering arrays [6]. The next important step in the realization of the GVD project was made in 2013 by deployment of an enlarged engineering array - the first stage of the first GVD-cluster, which comprised $72 \mathrm{OMs}$ arranged on three $345 \mathrm{~m}$ long strings and operated from April 2013 to February 2014 [1, 7]. In 2014 the second stage of the demonstration cluster, which comprised $112 \mathrm{OMs}$ arranged on five strings, as well as an instrumentation string with array calibration and environment monitoring equipment, was deployed. It was operated during 2014 in data taking and testing modes. One of the main goals of the operation in testing mode was to study the quality of in-situ calibration procedures. The performance and quality of these procedures have been verified by reconstructing position and intensity of a distant calibration laser source. The position of the laser source was reconstructed using the arrival times of photons detected by the PMTs, taking into account the timing calibration of the PMTs by LEDs. Results of the reconstruction were compared with the laser 


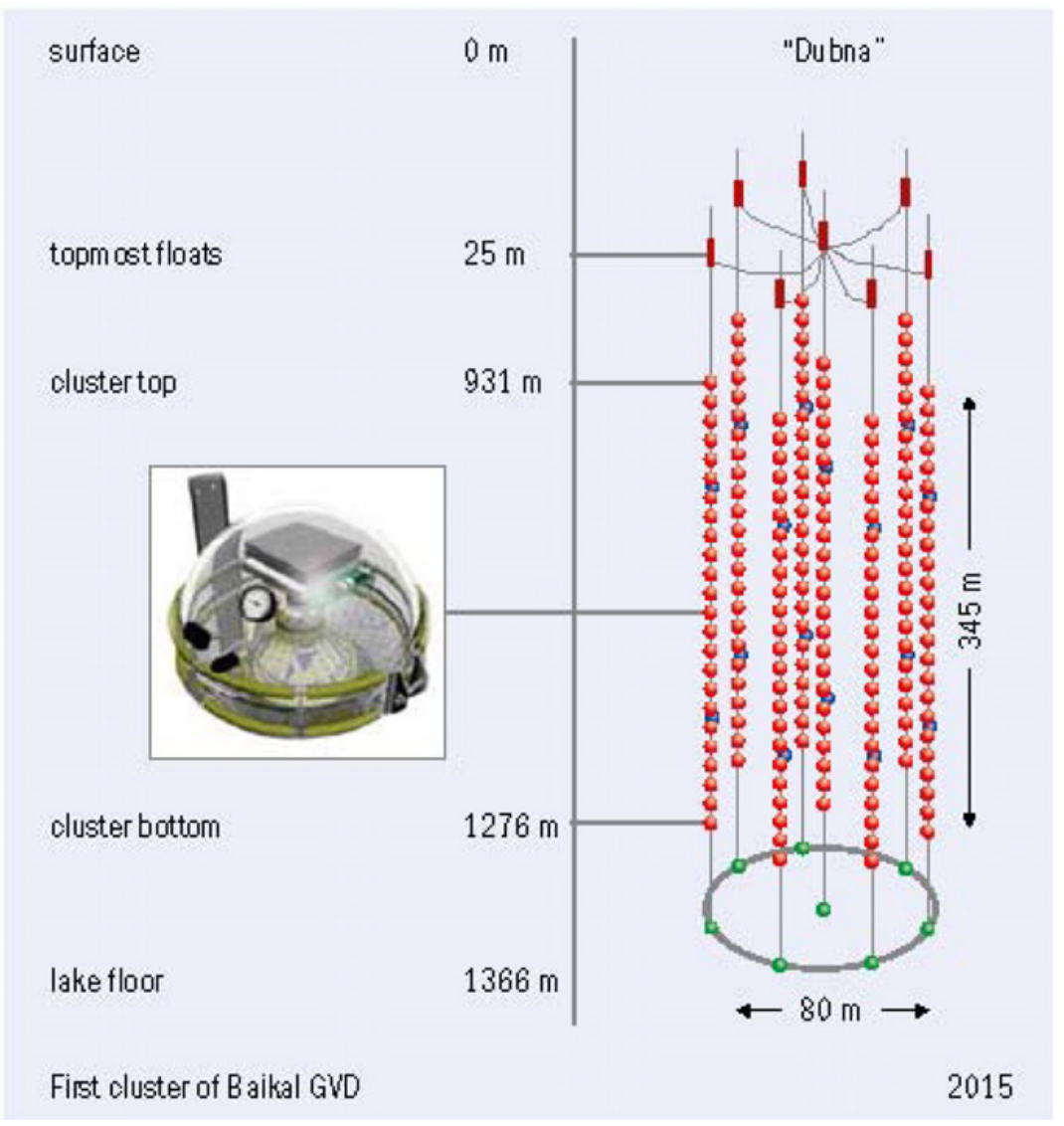

Figure 2. Schematic view of the "Dubna" cluster, the first cluster of the Baikal-GVD neutrino telescope.

coordinates obtained by the acoustic positioning system [8]. The obtained results prove the expected quality of the array calibration procedures.

\section{The first GVD-cluster}

In April 2015 the first demonstration cluster of Baikal-GVD named "Dubna" was deployed and commissioned in Lake Baikal (see Fig. 2). It encloses 1.7 Megatons of the clear waters of Lake Baikal. It comprises a total of 192 optical modules arranged at eight $345 \mathrm{~m}$ long strings, as well as an acoustic positioning system and an instrumentation string with equipment for array calibration and monitoring of environmental parameters. An artist's view of the cluster is shown in Fig. 2. Each string comprises 24 OMs spaced by $15 \mathrm{~m}$ at depths of $900 \mathrm{~m}$ to $1250 \mathrm{~m}$ below the surface. In 2015 seven side strings have been located at a reduced radius of $40 \mathrm{~m}$ around a central one (compared to $60 \mathrm{~m}$ for the baseline configuration). The reason is to increase the sensitivity to low-energy atmospheric muons and neutrinos which are used for array calibration. In the 2016, strings will be moved to the baseline distances.

Each optical module consists of a pressure-resistant glass sphere of $43.2 \mathrm{~cm}$ diameter which holds the OM electronics and the PMT which is surrounded by a high permittivity alloy cage for shielding it against the Earth magnetic field. A large photomultiplier tube Hamamatsu R7081-100 with a 10inch hemispherical photocathode and quantum efficiency up to $35 \%$ has been selected as light sensor. 

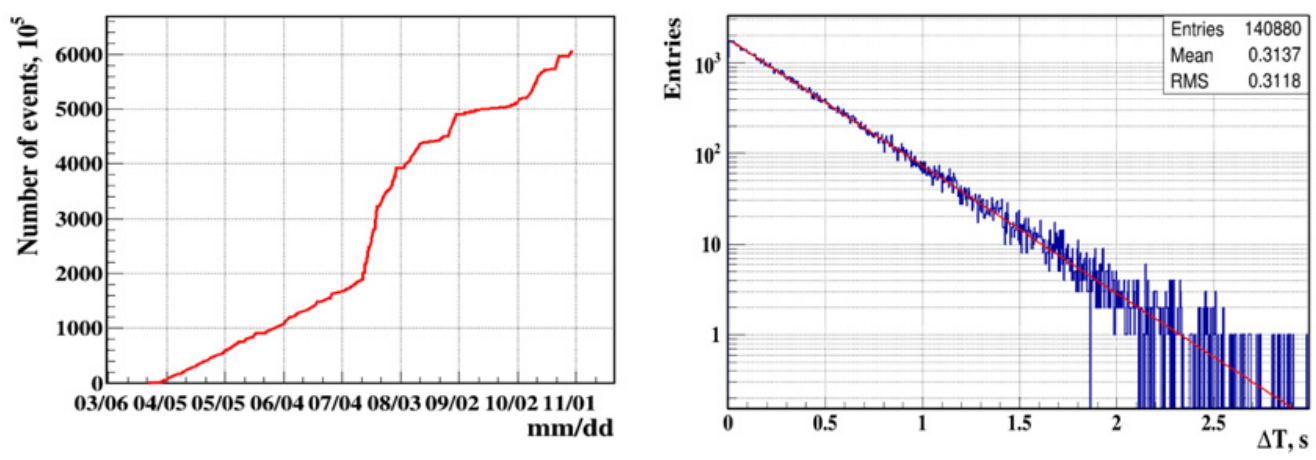

Figure 3. Left: Integrated number of recorded events since April 2015. Right: The time difference between subsequent events is shown for one run.

Besides the PMT, an OM comprises a high voltage power supply unit (HV), a fast two-channel preamplifier, and a controller. For time and amplitude calibration of the measuring channel, two LEDs are installed in the optical module. The OM controller is intended for HV control, monitoring of the PMT noise and for time and amplitude calibration [9].

The OMs along a string are combined in sections - the functional detection unit of the telescope [10]. Each section includes $12 \mathrm{OMs}$ and the central module $(\mathrm{CeM})$. PMTs signals from all OMs are transmitted through $90 \mathrm{~m}$ long coaxial cables to the CeM of the section, where they are digitized by custom-made ADC boards with $200 \mathrm{MHz}$ sampling rate. The waveform information from all measuring channels of the section is transferred to the Master board located in the CeM. The Master board provides readout of the ADC data, connection via local Ethernet to the cluster DAQ-center, control of the section operation and the section trigger logic. A request analyzer forms the section trigger request (local trigger) on the basis of channel requests $\mathrm{L}$ (low channel threshold, $\sim 0.3$ p.e.) and $\mathrm{H}$ (high threshold, $\sim 3$ p.e.) from $12 \mathrm{ADC}$ channels. This unit contains a programmable coincidence matrix $(12 \mathrm{H} \times 12 \mathrm{~L})$, which provides a simple way to generate the section trigger request. The basic trigger modes are: (A) coincidences of $>\mathrm{N}$ L-requests within a selectable time window ( $\mathrm{L}>\mathrm{N}$-trigger), or (B) coincidences of $\mathrm{L}$ and $\mathrm{H}$ requests from any neighbouring OMs within a section (L\&H-trigger). A request of the section trigger is transferred from the Master board through a string communication module (CoM) to the cluster DAQ-center, where a global trigger for all sections is generated. The cluster DAQ-center is located at the $30 \mathrm{~m}$ depth on central string. Data from the strings are transferred through DSL-modem Ethernet channel to the cluster center. The data transmission between the cluster DAQ-center and shore station is provided through optical fiber lines of about $6 \mathrm{~km}$ length.

The cluster has been operating from April 2015 in several testing and data taking modes. A total of $6.1 \times 10^{8}$ events has been recorded from April till November 2015. Figure 3 (left) gives an indication of the data taking efficiency during 2015. The change of the slope of the curve in Fig. 3 (left) is caused by the lowering of the used trigger thresholds in July. Shown in Fig. 3 (right) is the time di?erence between subsequent events for one run. The exponential behaviour of this distribution is consistent with the expectation for randomly distributed experimental events and illustrates the quality of the data. As an illustration, one event observed in June 2015 is shown in Fig. 4. The photon distribution of the event is consistent with the pattern of Cherenkov photons from a down-going high-energy muon.

The first cluster of Baikal-GVD has the potential to record astrophysical neutrinos with a flux value measured recently by IceCube [11, 12]. The search for high-energy neutrinos is based on the selection of cascade events generated by neutrino interactions in the sensitive volume of array. After applying an iterative procedure of vertex reconstruction followed by the rejection of hits contradicting 


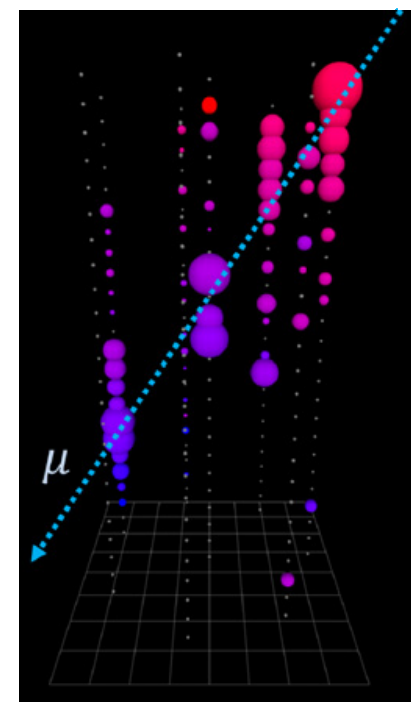

Figure 4. Event view of the high-energy down-going muon event observed in June 2015. Each sphere represents a OM. Colors represent the arrival times of the photons where red indicates early and blue late times. The size of the spheres is a measure for the recorded number of photoelectrons. The dotted line denotes the reconstructed track.

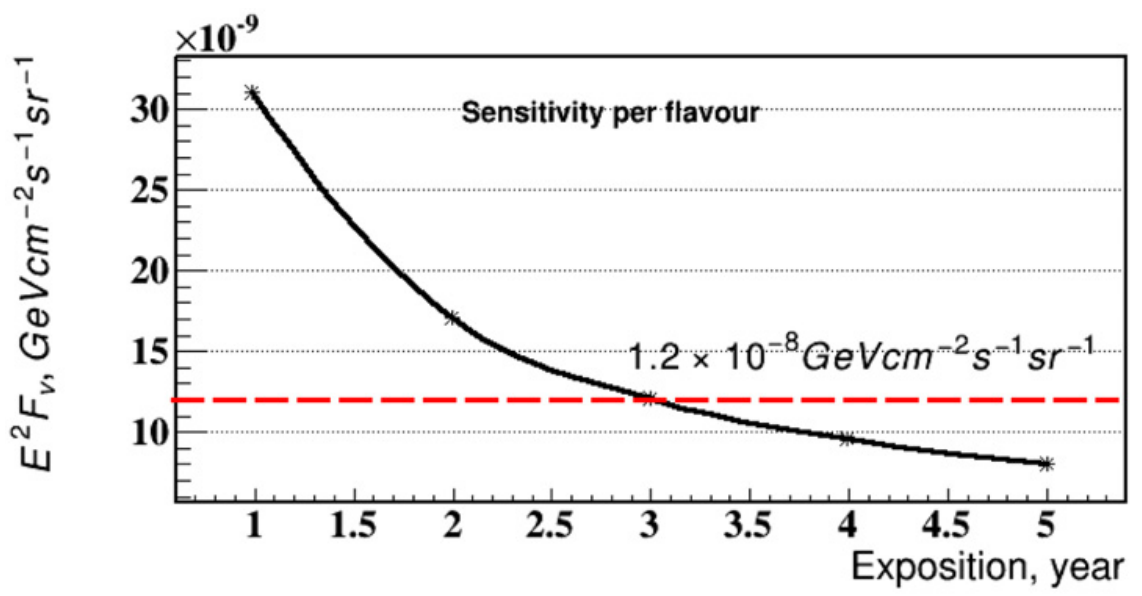

Figure 5. Cluster sensitivity for one flavor neutrino flux with an $\mathrm{E}^{-2}$ spectrum as function of the observation years. The long-dashed line indicates the one-flavor neutrino flux value obtained by IceCube.

the cascade hypothesis on each iteration stage, events with a final multiplicity of hit OMs $\mathrm{N}_{\text {hit }}>20$ are selected as high-energy neutrino events. The accuracy of shower direction reconstruction is about 4 degree (median value), which is substantially better than the 10-15 degree accuracy for IceCube [11]. We expect about one event per year with $\mathrm{E}_{\mathrm{sh}}>100 \mathrm{TeV}$ from an all-flavor astrophysical flux with the normalization $\mathrm{E}^{2} \mathrm{~F}=3.6 \cdot 10^{-8} \mathrm{GeV} \mathrm{cm}^{-2} \mathrm{~s}^{-1} \mathrm{sr}^{-1}$ in the cluster, compared to about 10 events in IceCube. The preliminary estimation of one cluster sensitivity for one flavor neutrino flux with an $\mathrm{E}^{-2}$ spectrum and flavor ration 1:1:1 for all flavor flux as function of the observation years is shown in Fig. 5. Three year exposition will result in a sensitivity corresponding to the level measured by IceCube. 
The baseline configuration of the Baikal-GVD cluster will comprise 288 OMs arranged at 8 strings. Each string will consist of 3 sections (12 OMs per section) arranged at depths from $700 \mathrm{~m}$ to $1250 \mathrm{~m}$. In 2016 the demonstration cluster will be upgraded to the baseline configuration with 8 new sections with a total of 96 OMs. By 2020, Baikal-GVD is set to consist of 8 baseline clusters covering a total volume of about $0.4 \mathrm{~km}^{3}$ (GVD phase-1).

\section{Conclusion}

The ambition of the Baikal collaboration is the construction of a $\mathrm{km}^{3}$-scale neutrino telescope the Gigaton Volume Detector in Lake Baikal. During the R\&D phase of the GVD project in 20082010, its basic elements - new optical modules, FADC readout units, underwater communications and trigger systems - have been developed, produced and tested in situ by long-term operating prototype strings. The prototyping phase of the GVD project has been performed since April 2011 and aimed at comprehensive in-situ tests of all elements and systems of the future detector. This phase has been concluded with deployment and commissioning of the first demonstration cluster of GVD in April 2015. For the years 2016-2020, the deployment of an array with about 2000 OMs is envisaged.

This work was supported by the Russian Found for Basic Research (grants 13-02-12221, 14-02-00175, 14-0200972).

\section{References}

[1] A. Avrorin et al., The prototyping/early construction phase of the BAIKAL-GVD project, NIM A742, 82

[2] A. Avrorin et al., Asp-15 - A stationary device for the measurement of the optical water properties at the NT200 neutrino telescope site, Nucl. Instr. Meth. A693, 186-194

[3] V. Aynutdinov et al., The prototype string for the km3-scale Baikal neutrino telescope, NIM A602, 227

[4] V. Aynutdinov et al., The BAIKAL neutrino experiment, NIM A626, 13

[5] V. Aynutdinov et al., The gigaton volume detector in Lake Baikal, NIM A639, 30

[6] A. Avrorin et al., Current status of the Baikal-GVD project, NIM A725, 23

[7] A. Avrorin et al., Status and recent results of the Baikal-GVD project, Phys. of Part. and Nucl. 46, 211

[8] A. Avrorin et al., A hydroacoustic positioning system for the experimental cluster of the cubickilometer-scale neutrino telescope at Lake Baikal, Instr. Exp.Tech. 56, 449

[9] A. Avrorin et al., The optical module of the Baikal-GVD neutrino telescope, these Proceedings

[10] A. Avrorin et al., Data acquisitions system of the NT1000 Baikal neutrino telescope, Instr. and Exp. Tech. 57, 262

[11] M. G. Aartsen et al., Evidence for high-energy extraterrestrial neutrinos at the IceCube detector, Science 342, 1242856

[12] M. G. Aartsen et al., Observation of high-energy astrophysical neutrinos in three years of IceCube data, Phys. Rev. Lett. 113, 101101 